\title{
(6) OPEN ACCESS \\ BCG vaccination at birth and early childhood hospitalisation: a randomised clinical multicentre trial
}

\author{
Lone Graff Stensballe, ${ }^{1}$ Signe Sørup, ${ }^{2}$ Peter Aaby, ${ }_{1}^{3}$ Christine Stabell Benn, ${ }^{4,5}$ \\ Gorm Greisen, ${ }^{6}$ Dorthe Lisbeth Jeppesen, ${ }^{7}$ Nina Marie Birk, ${ }^{7}$ Jesper Kjærgaard, ${ }^{8,9}$ \\ Thomas Nørrelykke Nissen, ${ }^{7}$ Gitte Thybo Pihl, ${ }^{10,11}$ Lisbeth Marianne Thøstesen, ${ }^{10,11}$ \\ Poul-Erik Kofoed, ${ }^{10,11}$ Ole Pryds, ${ }^{7}$ Henrik Ravn ${ }^{5,12}$
}

- Additional material is published online only. To view please visit the journal online (http://dx.doi.org/10.1136/ archdischild-2016-310760).

For numbered affiliations see end of article.

\section{Correspondence to} Dr Lone Graff Stensballe, The Child and Adolescent Clinic 4072, Juliane Marie Centret, Rigshospitalet, Copenhagen University Hospital,

Blegdamsvej 9, Copenhagen $\varnothing$ DK-2100, Denmark; Lone.graff. stensballe@regionh.dk

Received 25 February 2016 Revised 20 June 2016 Accepted 21 June 2016 Published Online First 21 July 2016
CrossMark

To cite: Stensballe $L G$,

Sørup S, Aaby $\mathrm{P}$, et al. Arch

Dis Child 2017;102:224-

231

\section{ABSTRACT}

Background The BCG vaccine is administered to protect against tuberculosis, but studies suggest there may also be non-specific beneficial effects upon the infant immune system, reducing early non-targeted infections and atopic diseases. The present randomised trial tested the hypothesis that BCG vaccination at birth would reduce early childhood hospitalisation in

Denmark, a high-income setting.

Methods Pregnant women planning to give birth at three Danish hospitals were invited to participate. After parental consent, newborn children were allocated to BCG or no intervention within 7 days of age.

Randomisation was stratified by prematurity. The primary study outcome was number of all-cause hospitalisations analysed as repeated events. Hospitalisations were identified using The Danish National Patient Register. Data were analysed by Cox proportional hazards models in intention-to-treat and per-protocol analyses.

Results 4184 pregnant women were randomised and their 4262 children allocated to BCG or no intervention. There was no difference in risk of hospitalisation up to 15 months of age; 2129 children randomised to BCG experienced 1047 hospitalisations with a mean of 0.49 hospitalisation per child compared with 1003 hospitalisations among 2133 control children (mean 0.47), resulting in a HR comparing BCG versus no BCG of 1.05 ( $95 \% \mathrm{Cl} 0.93$ to 1.18$)$ (intention-to-treat analysis). The effect of BCG was the same in children born at term (1.05 (0.92 to 1.18)) and prematurely (1.07 (0.63 to 1.81), $p=0.94)$. The effect was also similar in the two sexes and across study sites. The results were essentially identical in the per-protocol analysis and after adjustment for baseline characteristics. Conclusions BCG vaccination at birth did not reduce the risk of hospitalisation for somatic acquired disease until 15 months of age in this Danish study population. Trial registration number NCT01694108, results.

\section{BACKGROUND}

BCG, primary prophylaxis against tuberculosis, is among the most widely used vaccines globally. ${ }^{1}$ Randomised controlled trials indicate that the live attenuated BCG vaccine against tuberculosis may have beneficial 'non-specific' or 'heterologous' effects on the infant immune system, reducing nontuberculosis neonatal mortality in low-income settings by reducing severe early infection. ${ }^{2}{ }^{3}$ The association between BCG and childhood morbidity,

\section{What is already known on this topic?}

- BCG is the primary prophylaxis against tuberculosis.

- BCG is among the most widely used vaccines globally.

- There may be non-specific beneficial effects of neonatal BCG vaccination upon the infant immune system, reducing early infections and atopic disease.

\section{What this study adds?}

BCG vaccination did not reduce the risk of hospitalisation for acquired somatic disease during the first 15 months of life in a Danish study population.

primarily atopic disease has been studied with conflicting results. ${ }^{4}$ The only randomised trial so far among 121 newborns with high risk of atopy found BCG to be associated with a significant reduction in the use of medication for eczema. ${ }^{5}$ From 1979, routine BCG vaccination at school start was gradually discontinued in Denmark due to low prevalence of tuberculosis. If BCG has beneficial non-specific effects on health also in highincome settings, stopping routine BCG may have deprived children of these beneficial effects. We therefore conducted a large-scale randomised trial in Denmark to test the effect of BCG given at birth on childhood morbidity, including hospitalisation, infections, atopic disease, growth, development and immunological indicators. The present paper presents the effect of BCG on the primary outcome, all-cause hospitalisation from birth to 15 months of age. We hypothesised that BCG vaccination at birth would reduce all-cause hospitalisation by $20 \%$.

\section{METHODS}

Setting, study population

The procedures of The Danish Calmette Study were published in details elsewhere. ${ }^{6}$ Briefly, pregnant women planning to give birth at three Danish 
hospitals were invited. Inclusion criteria were gestational age of $32+$ weeks, birth weight of $1000+$ grams and a signed informed consent from the parents. Exclusion criteria were maternal intake of immunomodulating medicine during pregnancy, signs of severe illness or major malformation in the infant and no Danish-speaking parent due to concerns about language barrier. Newborns were allocated 1:1 to standard BCG vaccination with the Danish strain 1331, or no intervention within 7 days of birth. The children were allocated 1:1 in permuting blocks of 2-4-6 using an online system. Since in prior studies the strongest nonspecific effects on mortality have been seen among the smallest newborns, ${ }^{2} 3$ the allocation was stratified by prematurity defined as gestational age $<37$ weeks. The allocation was planned stratified also by sex and study site; however, by programme error the allocation was stratified only by prematurity. Parents were not blinded to allocation since the local inflammatory reaction caused by BCG vaccination could not be mimicked, but the parents were asked not to disclose if the child had received BCG to keep study staff blinded during the data collection.

\section{Follow-up}

The primary outcome was all-cause hospitalisations. Since the measles-mumps-rubella vaccine (MMR) may also have nonspecific effects, follow-up was censored at 15 months of age where MMR is scheduled in the Danish national vaccination programme. $^{78}$

All Danish residents have a unique identification number, which was used to obtain information from The Danish National Patient Register on acquired, somatic all-cause hospitalisation, including dates of admission and discharge and categorised by the International Classification of Diseases V.10 (ICD10) diagnosis codes. ${ }^{9}{ }^{10}$ Outpatient and emergency department hospital contacts not leading to admission, and hospitalisations starting before or at the date of randomisation were excluded. Since the study aimed to examine if BCG reduced acquired somatic morbidity, diagnostic codes due to administrative procedures, admission to the maternity ward in connection with the delivery, congenital and birth-related conditions, psychiatric disease, and injuries were excluded (ICD10-specification in the online supplement).

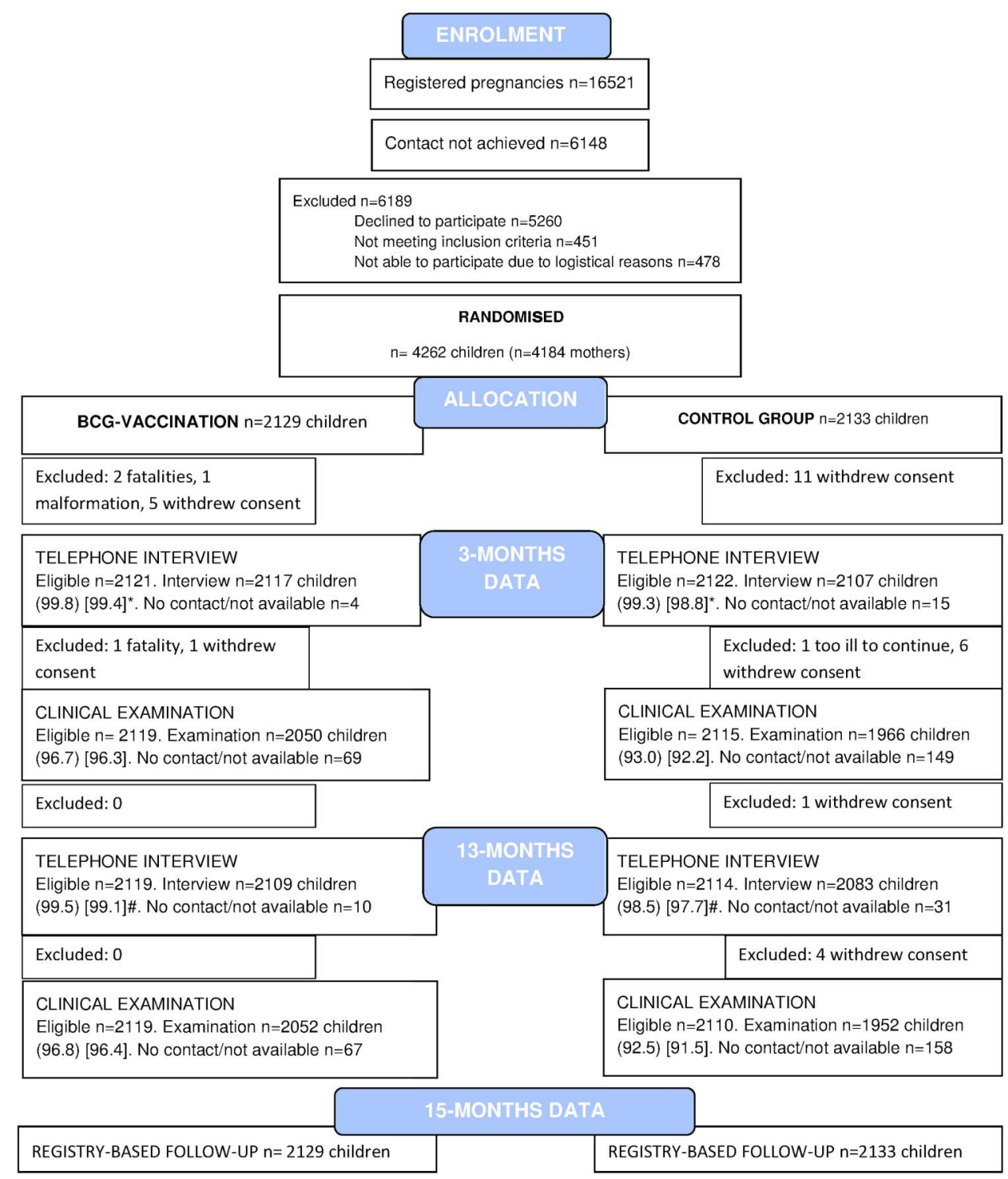

Figure 1 Flow chart. Data collection of The Danish Calmette Study, by telephone interviews, clinical examinations and health registers. Percentages in parentheses (): Percentage among the eligible children. Percentages in square brackets []: Percentage among all randomised children. *Interviews conducted between child age 2-4 months: BCG 98.1\% (2089/2129), control 96.8\% (2064/2133). \#Interviews conducted between child age 10-14 months: BCG 97.9\% (2084/2129), control 96.2\% (2052/2133). 
Furthermore, follow-up consisted of telephone interviews and clinical examinations at 3 months and 13 months of age; secondary outcomes and adverse events will be published separately.

\section{Power calculation}

Sample size estimates were based on $95 \%$ CIs and a $90 \%$ power. Twenty per cent of the child population in Denmark was expected to be hospitalised during the first 15 months of life. ${ }^{11}$ To detect a $20 \%$ reduction in hospitalisations during the first 15 months of life, 3972 newborns needed to be included. We expected a very small loss to follow-up in the registry-based studies and aimed to include approximately 4300 newborns.

\section{Statistics}

Cox proportional hazards models were used to estimate the HR of all-cause hospitalisation between the BCG and control groups. Adjustment for recurrent hospitalisations for each child was done using robust SEs for the estimated HRs. The results are presented as HRs with 95\% CIs and corresponding $\mathrm{p}$ values. The children were censored at migration, 15 months of age or death, whichever came first. The analyses were stratified by prematurity in accordance with the randomisation procedure. In the main 'intention-to-treat (ITT) analysis' hospitalisations within the period from randomisation to 15 months of age were analysed according to randomisation group. We also conducted a 'per-protocol (PP) analysis' in which children who did not follow the allocation were excluded and hospitalisations from time since vaccination for the BCG group and time since randomisation for controls was included. Curves for mean number of hospitalisations by randomisation arm as a function of time since randomisation were estimated using the Nelson-Aalen method. Further, we tested the effect of BCG on time to first hospitalisation.

\section{Secondary analyses}

Using Cox models, we tested if adjustment for baseline characteristics changed the estimates. Since previous studies indicate that the non-specific effects of one vaccine may be modified by a subsequent different vaccination, ${ }^{12}$ follow-up was divided into 0-2 months (0-89 days) while the children had received BCG or nothing, and 3-15 months (90-456 days) when the children should have received one to three doses of diphtheria, tetanus, acellular pertussis, polio, Haemophilus influenzae type b and Streptococcus pneumonia vaccine according to the routine vaccination programme. ${ }^{8}$

We also estimated potential effect modification by caesarean section, administration of antibiotics to the mother during delivery, birth weight $<2500 \mathrm{~g}$, $\geq$ one sibling, atopic disposition, vaccination $\leq 2$ days of age, maternal BCG vaccination and by the two vaccine batches used in the study. The analysis by batch was adjusted for calendar months.

All analyses were performed using Stata V.13 (StataCorp LP, Texas, USA).

\section{RESULTS}

\section{Randomisation and baseline}

At the three hospitals 16521 pregnant women were invited. No response was given by 6148 families, and 6189 families were excluded primarily because they declined participation after being informed verbally about the study (figure 1). Four thousand one hundred and eighty-four pregnant women were randomised and their 4262 children allocated to BCG or no intervention (figure 1). Baseline characteristics with potential influence on childhood morbidity did not differ between the two allocation groups except for ethnicity other than Danish and smoking, which were more common in families of control children (table 1).

The study population was characterised by a large proportion of highly educated parents, with a high prevalence of atopic diseases.

\section{Treatment assignments and crossovers}

Two thousand one hundred and twenty-nine children were randomised to receive BCG and 2133 children to the control group. Eleven children randomised to the BCG group did not receive the $B C G$ vaccine and 36 children randomised to the control group received the BCG vaccine on their own initiative leaving 2118 children allocated to BCG and BCG-vaccinated groups and 2097 children randomised to control and not vaccinated groups in the PP analysis.

Table 1 Baseline characteristics by allocation among 4184 Danish mothers in The Danish Calmette Study

\begin{tabular}{|c|c|c|}
\hline & $\begin{array}{l}\text { BCG (N=2095) } \\
n(\% *)(N A)\end{array}$ & $\begin{array}{l}\text { Control } \\
\text { (N=2089) } \\
\text { n (\%) (NA) }\end{array}$ \\
\hline Male sext & $1092(52.1)(0)$ & $1104(52.9)(0)$ \\
\hline Prematurity (GA<37 weeks) & $61(2.9)(0)$ & $60(2.9)(0)$ \\
\hline Randomisation site & (0) & (0) \\
\hline Rigshospitalet & $751(35.9)$ & $780(37.3)$ \\
\hline Hvidovre & $730(34.8)$ & $719(34.4)$ \\
\hline Kolding & $614(29.3)$ & $590(28.4)$ \\
\hline Caesarean section & $411(19.6)(0)$ & $442(21.2)(0)$ \\
\hline Antibiotics during delivery $¥$ & $333(15.9)(0)$ & $358(17.1)(0)$ \\
\hline Singletons & $2061(98.4)(0)$ & $2046(97.9)(0)$ \\
\hline Twins & $34(1.6)$ & $42(2.0)$ \\
\hline Triplets & 0 & $1(0.0)$ \\
\hline Birth weight in grams (mean \pm SD) & $3519 \pm 493(0)$ & $3523 \pm 494(0)$ \\
\hline Child $<1$ day of age at randomisation & $1006(48.0)(0)$ & $1000(47.9)(0)$ \\
\hline $\begin{array}{l}\text { Maternal age in years at birth } \\
(m e a n \pm S D)\end{array}$ & $32.0 \pm 4.6(0)$ & $31.9 \pm 4.4(0)$ \\
\hline $\begin{array}{l}\geq 1 \text { parent of ethnicity other than } \\
\text { Danish }\end{array}$ & $376(18.1)(14)$ & $458(22.1)(15)$ \\
\hline Maternal education & (7) & (6) \\
\hline No higher education & $460(22.0)$ & $435(20.9)$ \\
\hline Short/medium higher education & $935(44.8)$ & $939(45.1)$ \\
\hline Long higher education & $693(33.2)$ & $709(34.0)$ \\
\hline Parents living together & $1984(94.9)(4)$ & $1984(95.0)(0)$ \\
\hline Mother BCG vaccinated & $364(17.6)(27)$ & $353(17.2)(41)$ \\
\hline$\geq 1$ older sibling & $887(42.4)(1)$ & $843(40.4)(2)$ \\
\hline \multicolumn{3}{|l|}{ Atopic disposition§ } \\
\hline Maternal atopic disease & $839(41.6)(80)$ & $809(40.3)(83)$ \\
\hline Paternal atopic disease & $717(38.2)(217)$ & 708 (37.7) (209) \\
\hline Parental atopic disease & $1265(65.7)(168)$ & $\begin{array}{l}1243(64.1) \\
(151)\end{array}$ \\
\hline Siblings with atopic disease & 275 (13.3) (30) & $252(12.2)(23)$ \\
\hline \multicolumn{3}{|l|}{ Smoking during pregnancy } \\
\hline Maternal & $203(9.7)(1)$ & $212(10.2)(1)$ \\
\hline Paternal & $388(18.9)(38)$ & $458(22.2)(31)$ \\
\hline
\end{tabular}

*Percentage among families where the information was available. tThe sex of the first child in multiple births.

$\ddagger$ Antibiotics to the mother during elective caesarean section were administered after disconnection of the umbilical cord at the Kolding site and before the umbilical cord was disconnected at Rigshospitalet and Hvidovre sites.

$\S$ Atopic disposition defined as physician-diagnosed atopic eczema, asthma, allergic rhinoconjunctivitis or food allergy.

$\mathrm{GA}$, gestational age; NA, not available (number of families without information on the specified variable). 
Table 2 Number of hospitalisations among 4262 Danish children by allocation to BCG vaccination at birth or control group (no intervention)

\begin{tabular}{|c|c|c|c|c|c|c|c|c|}
\hline $\begin{array}{l}\text { Intention-to-treat } \\
\text { analysis* }\end{array}$ & $\begin{array}{l}\text { Allocated to BCG. } \\
\text { Number of } \\
\text { hospitalisations } \\
\text { (nt) (mean } \ddagger \text { ) }\end{array}$ & $\begin{array}{l}\text { Allocated to } \\
\text { control. } \\
\text { Number of } \\
\text { hospitalisations } \\
\text { (nt) (mean } \ddagger)\end{array}$ & $\begin{array}{l}\text { HR, } \\
\text { crude }\end{array}$ & $95 \% \mathrm{Cl}$ & $\begin{array}{l}\mathrm{p} \text { Value of effect } \\
\text { modification } \\
\text { between the } \\
\text { groups }\end{array}$ & $\begin{array}{l}\text { HR, } \\
\text { adjusted§ }\end{array}$ & $95 \% \mathrm{Cl}$ & $\begin{array}{l}p \text { Value of effect } \\
\text { modification } \\
\text { between the } \\
\text { groups }\end{array}$ \\
\hline All $n=4262$ & 1047 (2129) (0.49) & $1003(2133)(0.47)$ & 1.05 & 0.93 to 1.18 & & 1.05 & 0.93 to 1.20 & \\
\hline Prematurity & & & & & 0.94 & & & 0.14 \\
\hline Premature $n=144$ & $62(71)(0.87)$ & $59(73)(0.81)$ & 1.07 & 0.63 to 1.81 & & 1.56 & 0.91 to 2.66 & \\
\hline Mature $n=4118$ & 985 (2058) (0.48) & $944(2060)(0.46)$ & 1.05 & 0.92 to 1.18 & & 1.03 & 0.91 to 1.17 & \\
\hline Sex & & & & & 0.44 & & & 0.48 \\
\hline Male $n=2241$ & $604(1104)(0.55)$ & 570 (1137) (0.50) & 1.09 & 0.93 to 1.29 & & 1.09 & 0.93 to 1.29 & \\
\hline Female $n=2021$ & $443(1025)(0.43)$ & 433 (996) (0.44) & 0.99 & 0.83 to 1.20 & & 1.00 & 0.82 to 1.21 & \\
\hline Study site & & & & & 0.20 & & & 0.12 \\
\hline Rigshospitalet $n=1567$ & $383(764)(0.50)$ & 391 (803) (0.49) & 1.04 & 0.85 to 1.28 & & 1.02 & 0.82 to 1.27 & \\
\hline Hvidovre $n=1470$ & 451 (738) (0.61) & $384(732)(0.53)$ & 1.16 & 0.96 to 1.41 & & 1.21 & 0.99 to 1.47 & \\
\hline Kolding $n=1225$ & $213(627)(0.34)$ & $228(598)(0.38)$ & 0.88 & 0.69 to 1.12 & & 0.87 & 0.68 to 1.11 & \\
\hline $\begin{array}{l}\text { Per-protocol } \\
\text { analysis** }\end{array}$ & $\begin{array}{l}\text { BCG vaccinated. } \\
\text { Number of } \\
\text { hospitalisations } \\
\text { (nt) (mean } \ddagger)\end{array}$ & $\begin{array}{l}\text { Control group. } \\
\text { Number of } \\
\text { hospitalisations } \\
\text { (nt) (mean } \ddagger)\end{array}$ & HR & $95 \%-\mathrm{Cl}$ & $\begin{array}{l}p \text { Value of effect } \\
\text { modification } \\
\text { between the } \\
\text { groups }\end{array}$ & $\begin{array}{l}\text { HR, } \\
\text { adjusted§ }\end{array}$ & $95 \%-\mathrm{Cl}$ & $\begin{array}{l}p \text { Value of effect } \\
\text { modification } \\
\text { between the } \\
\text { groups }\end{array}$ \\
\hline All $n=4215$ & $1044(2118)(0.49)$ & 979 (2097) (0.47) & 1.06 & 0.93 to 1.19 & & 1.06 & 0.93 to 1.20 & \\
\hline Prematurity & & & & & 0.90 & & & 0.13 \\
\hline Premature $n=143$ & $62(71)(0.87)$ & $57(72)(0.79)$ & 1.09 & 0.64 to 1.87 & & 1.60 & 0.92 to 2.77 & \\
\hline Mature $n=4071$ & $982(2047)(0.48)$ & $922(2025)(0.45)$ & 1.05 & 0.93 to 1.20 & & 1.04 & 0.91 to 1.18 & \\
\hline Sex & & & & & 0.35 & & & 0.39 \\
\hline Male $n=2214$ & 604 (1096) (0.55) & 554 (1118) (0.50) & 1.11 & 0.95 to 1.31 & & 1.11 & 0.94 to 1.31 & \\
\hline Female $n=2000$ & $440(1022)(0.43)$ & 425 (979) (0.43) & 0.99 & 0.82 to 1.19 & & 1.00 & 0.82 to 1.21 & \\
\hline Study site & & & & & 0.28 & & & 0.17 \\
\hline Rigshospitalet $n=1543$ & $383(761)(0.50)$ & 379 (782) (0.49) & 1.05 & 0.85 to 1.30 & & 1.03 & 0.83 to 1.28 & \\
\hline Hvidovre $n=1456$ & 448 (734) (0.61) & $379(722)(0.53)$ & 1.16 & 0.96 to 1.41 & & 1.20 & 0.98 to 1.46 & \\
\hline Kolding $n=1215$ & $213(623)(0.34)$ & $221(593)(0.37)$ & 0.91 & 0.71 to 1.15 & & 0.89 & 0.70 to 1.14 & \\
\hline
\end{tabular}

Intention-to-treat and per-protocol analyses.

${ }^{*}$ All children randomised were followed from randomisation to 15 months of age and analysed according to randomisation group.

tNumber of children.

¥Mean hospitalisation per child.

$\S$ Adjusted for baseline characteristics (sex, prematurity, study site, caesarean section, antibiotics during delivery, multiple birth, birth weight, child age at randomisation, maternal age parental ethnicity ( $\geq 1$ parent of ethnicity other than Danish), maternal education, parents living together, maternal BCG (by maternal recall), siblings, maternal atopy and maternal smoking during pregnancy).

TPrematurity defined as birth before 37 weeks of gestation.

${ }^{* *}$ Children having received the BCG vaccine but randomised to control group, or vice versa were excluded. The remaining children were followed from time since vaccination for the BCG group and time since randomisation for control children.

Primary outcome: risk of all-cause hospitalisation

The rate of follow-up was 100\% (figure 1). Until 15 months of age, the 2129 children randomised to BCG experienced a total of 1047 hospitalisations, the mean hospitalisation per child being 0.49; compared with 1003 hospitalisations among 2129 children randomised to control, the mean hospitalisation per control child being 0.47 . Hence, there was no difference in number of hospitalisations from randomisation to 15 months of age in the ITT analysis (table 2 and figure 2).

There was no effect modification by prematurity, sex or study site (table 2); however, the rate of hospitalisation was lower at the Kolding site where short acute admissions were classified as outpatient consultations. The results were essentially identical in the PP analysis (table 2). If the outcome was defined as time to first hospitalisation instead of recurrent hospitalisations, the HRs were 1.01 (0.91 to 1.13 ) (ITT) and 1.02 (0.92 to 1.14$)$ (PP), respectively. The inclusion in the analysis of children from multiple births as clusters did not change the estimates (data not shown).

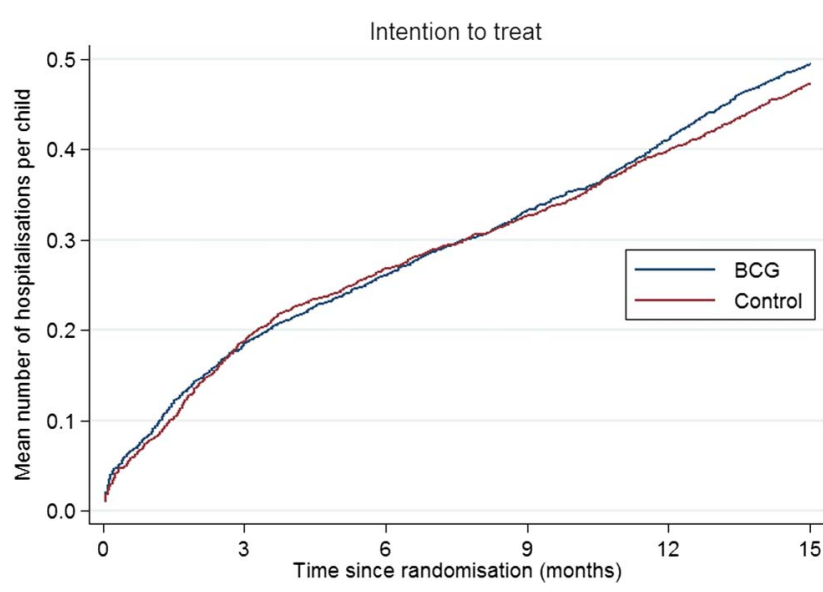

Figure 2 Estimated mean number of all-cause hospitalisations per child for children randomised to BCG or no BCG. 


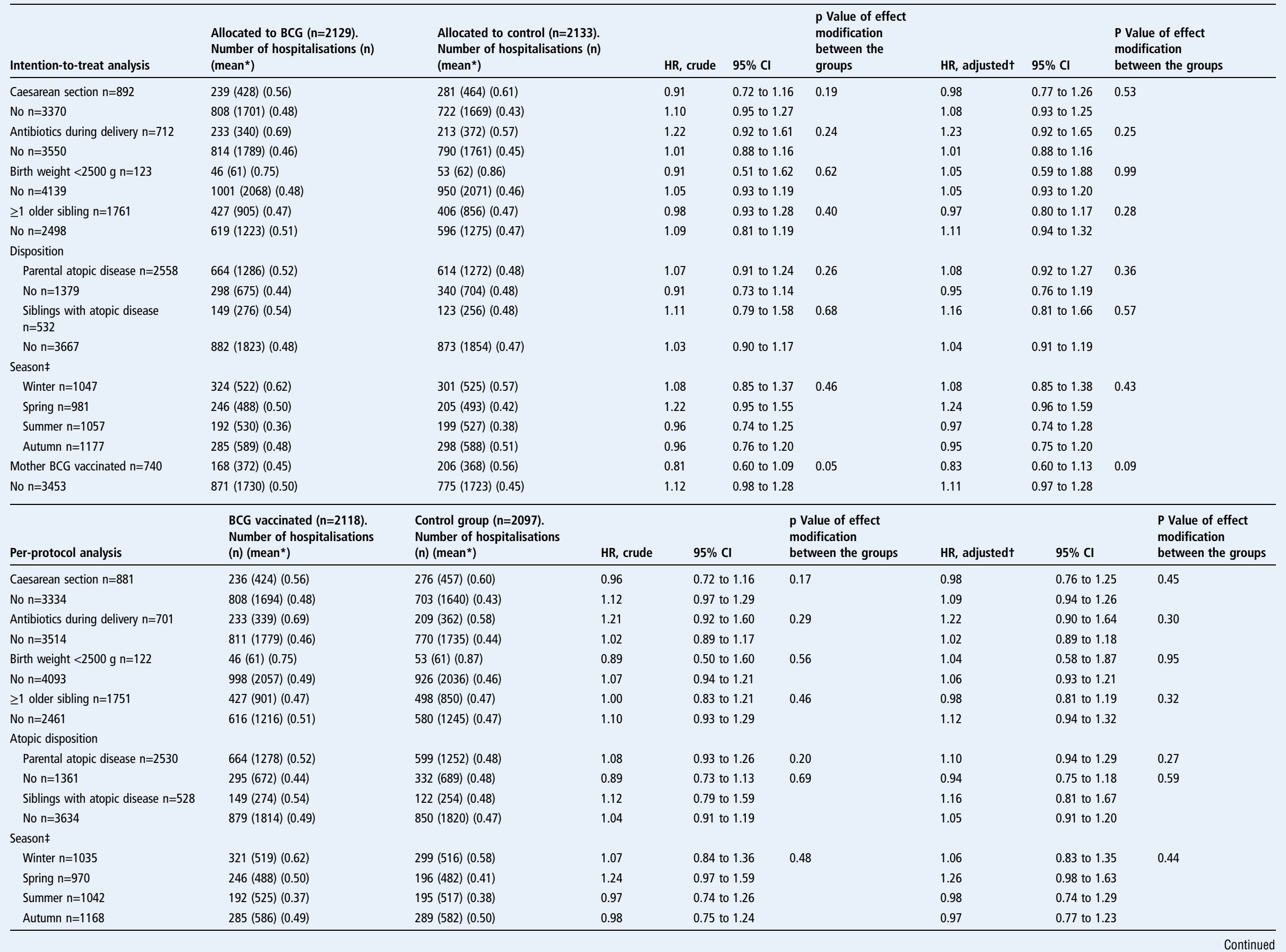




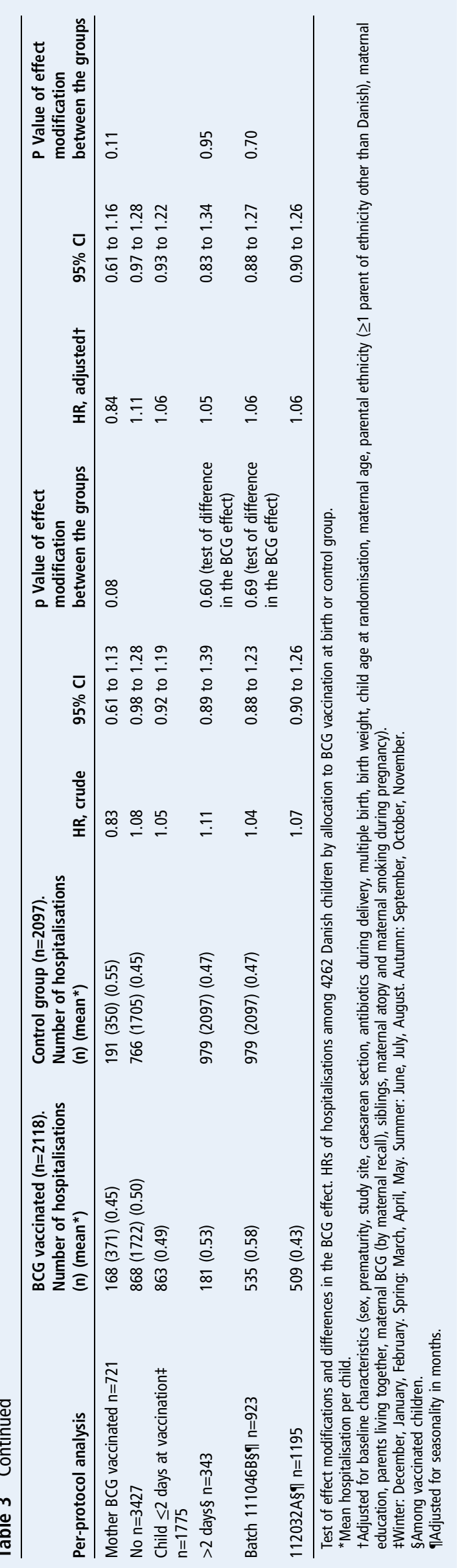

Secondary analyses

Adjustment for all baseline characteristics essentially did not change the estimates (see table 2 and 3), the HR of hospitalisation was 1.05 (0.93 to 1.20) for children randomised to BCG. For children randomised to BCG, the risk of hospitalisation was 0.98 (0.83 to 1.16$)$ until 3 months and 1.09 (0.94 to 1.27 ) after 3 months of age. No statistically significant effect modification by caesarean section, administration of antibiotics to the mother during vaginal delivery, birth weight $<2500 \mathrm{~g}, \geq 1$ sibling, atopic disposition, age at vaccination $\leq 2$ days, maternal BCG vaccination and BCG vaccine batch was observed (table 3 ). However, a tendency towards protection against hospitalisation was observed among BCGvaccinated children whose mother was also BCG vaccinated (table 3).

\section{DISCUSSION}

We hypothesised that BCG vaccination at birth would have nonspecific beneficial effects and reduce overall childhood hospitalisation for somatic acquired disease (injuries excluded) from birth to 15 months of age by $20 \%$ in the high-income setting of Denmark. We were not able to confirm this.

\section{Strengths and weaknesses}

The strengths of the present study lie in the randomised clinical multicentre trial design with adequate power. Data on the primary study outcome, all-cause hospitalisation, were collected independently of the study, decreasing the risk of bias. All participants could be followed up through the public registers.

The study may be limited by our choice of all-cause hospitalisation as primary outcome, which was chosen because of its potentially high impact on public health and because this outcome included both hospitalisation-requiring infections and severe manifestations of atopic disease. Given the high incidence of hospitalisations, this outcome, however, may not have been specific enough to detect a potential beneficial immune-training effect of BCG.

\section{Comparison with other studies}

In low-income settings, two randomised controlled trials among low birthweight children found BCG to reduce non-tuberculosis mortality until 6 months of age, in particular neonatal sepsis and respiratory infections. ${ }^{2} 3$ Recent immunological studies have provided a potential mechanism by showing that BCG induces trained innate immunity through epigenetic reprogramming of monocytes, ${ }^{13}{ }^{14}$ which was still present 12 months after BCG. ${ }^{13} 15$ In Guinean babies, BCG was associated with increased responses to heterologous innate stimulation. ${ }^{16}$

There may be other explanations for the lack of effect of BCG on all-cause hospitalisation in Denmark.

First, in a system with free healthcare, hospitalisation for acquired somatic disease may not represent a sufficiently specific measure of disease. A high level of parental concern in combination with a low professional threshold for hospitalisation of young infants is likely to have inflated the hospitalisation rate. The rate of all-cause hospitalisation was 50\% higher than expected.

Second, there are obvious differences in exposure to infection between Denmark and the low-income settings where the beneficial non-specific effects of BCG on mortality have been observed. The majority of children in the present study had no 
siblings, thus, presumably the exposure to infections was limited until they started day care around 1 year of age.

Third, we previously found that maternal immunity may also be of importance to the non-specific response to measles vaccination in the child; ${ }^{17}$ we therefore also asked whether the mothers in The Danish Calmette Study had been BCG vaccinated. In low-income settings the majority of mothers will have been BCG vaccinated, whereas in our study only $17 \%$ of the mothers had been BCG vaccinated, because BCG was discontinued in the early 1980s. That early exposure may be of importance to the child's response to BCG was also indicated by two studies comparing cytokine responses after BCG in UK and Malawi infants, and finding significant differences, which were ascribed to exposure very early, in utero, or within the first few months of life. ${ }^{18} 19$ Further, it has been shown that foetal T helper cells can be sensitised to mycobacterial purified protein derivative in utero. ${ }^{20}$ In agreement with this, in a preplanned secondary analysis of hospitalisations for infection within the present trial, a significant beneficial effect of BCG among children of BCG-vaccinated mothers was observed (personal communication, Stensballe LG, Greisen G, Jeppesen DL, et al. The effect of BCG vaccination at birth on risk of hospitalization for infection in Denmark. A randomized clinical multicenter trial. 2015. Unpublished work). If maternal exposure to BCG or mycobacteria is essential for the development of beneficial nonspecific effects of BCG, this may explain the beneficial effect of BCG observed in low-income countries but not overall in the present study.

Fourth, genetics differ between the populations of West Africa and Denmark; $;^{21-23}$ however, the influence of this is not clear and it should be noted that others have found evidence of beneficial effects of BCG in Denmark. ${ }^{24}$

\section{CONCLUSIONS}

BCG vaccine at birth did not decrease the risk of hospitalisation for somatic acquired disease until 15 months of age in our highincome setting.

\section{Author affiliations}

${ }^{1}$ The Child and Adolescent Clinic 4072, Juliane Marie Centret, Rigshospitalet, Copenhagen University Hospital, Copenhagen $\varnothing$, Denmark

${ }^{2}$ Research Center for Vitamins and Vaccines (CVIVA), Bandim Health Project, Statens Serum Institut, Copenhagen S, Denmark

${ }^{3}$ Bandim Health Project, Statens Serum Institut, Copenhagen S, Denmark

${ }^{4}$ Research Center for Vitamins and Vaccines (CVIVA), Statens Serum Institut, Copenhagen S, Denmark

${ }^{5}$ OPEN, Institute of Clinical Research, University of Southern Denmark/Odense University Hospital, Denmark

${ }^{6}$ The Neonatal Department, Juliane Marie Centret, Rigshospitalet, Copenhagen University Hospital, Copenhagen $\varnothing$, Denmark

${ }^{7}$ Department of Paediatrics, 460, Copenhagen University Hospital, Hvidovre, Hvidovre, Denmark

8Research Unit Womens' and Childrens' Health, The Child and Adolescent Clinic 4072, Juliane Marie Centret, Rigshospitalet, Copenhagen University Hospital, Copenhagen $\varnothing$, Denmark

${ }^{9}$ Denmark Copenhagen University Hospital, Copenhagen $\varnothing$, Denmark

${ }^{10}$ Department of Paediatrics, Kolding Hospital, Kolding, Denmark

${ }^{11}$ Institute of Regional Health Research, University of Southern Denmark, Denmark

${ }^{12}$ Research Center for Vitamins and Vaccines (CVIVA), Bandim Health Project,

Statens Serum Institut, Copenhagen S, Denmark
}

Acknowledgements The authors thank the families and children for their participation, and The Danish Calmette Study staff for their commitment. The authors also thank the Data Safety Monitoring Board members Frank Shann, Professor of Paediatric Intensive Care Medicine, Melbourne, Per Kragh Andersen, Professor of Biostatistics, Copenhagen, and Jørn Olsen, Professor of Epidemiology, Aarhus (chair).
Contributors LGS conceived the idea and is the guarantor of the study. LGS, GG, $\mathrm{DLJ}, \mathrm{P}-\mathrm{EK}, \mathrm{OP}$ supervised the collection of data by telephone interviews and clinical examinations and NMB, JK, TNN, GTP, LMT participated in this data collection. LGS, GG, P-EK, OP, CSB and PA constituted the Trial Steering Committee. LGS, HR and SS obtained the register-based data. HR, LGS and SS carried out data management. HR and LGS analysed the data. LGS drafted the manuscript. All authors read and approved the final manuscript.

Funding The study was funded by the three involved hospitals and by the Danish National Research Foundation (DNRF108).

Competing interests None declared.

Ethics approval The trial was approved by the Committees on Biomedical Research Ethics (J.no. H-3-2010-087), the Danish Data Protection Board (J.no. 2009-41-4141), and the Danish Medicines Agency (J.no. 2612-4356. EudraCT 2010-021979-85. Protocol 2009-323).

Provenance and peer review Not commissioned; externally peer reviewed.

Data sharing statement $A$ check of the validity of the data and analyses for this study can be arranged with the corresponding author. Request for access to a data copy should be directed to the Trial Steering Committee.

Open Access This is an Open Access article distributed in accordance with the Creative Commons Attribution Non Commercial (CC BY-NC 4.0) license, which permits others to distribute, remix, adapt, build upon this work non-commercially, and license their derivative works on different terms, provided the original work is properly cited and the use is non-commercial. See: http://creativecommons.org/ licenses/by-nc/4.0/

\section{REFERENCES}

1 Ottenhoff TH, Kaufmann SH. Vaccines against tuberculosis: where are we and where do we need to go? PLoS Pathog 2012;8:e1002607.

2 Aaby $P$, Roth $A$, Ravn $\mathrm{H}$, et al. Randomized trial of $B C G$ vaccination at birth to low-birth-weight children: beneficial nonspecific effects in the neonatal period? J Infect Dis 2011;204:245-52.

3 Biering-Sørensen S, Aaby P, Napirna BM, et al. Small randomized trial among low-birth-weight children receiving bacillus Calmette-Guérin vaccination at first health center contact. Pediatr Infect Dis J 2012;31:306-8.

4 Arnoldussen DL, Linehan M, Sheikh A. BCG vaccination and allergy: a systematic review and meta-analysis. J Allergy Clin Immunol 2011;127: 246-53, 253.

5 Steenhuis TJ, van Aalderen WM, Bloksma N, et al. Bacille-Calmette-Guerin vaccination and the development of allergic disease in children: a randomized, prospective, single-blind study. Clin Exp Allergy 2008;38:79-85.

6 Thøstesen LM, Nissen TN, Kjærgaard J, et al. Bacillus Calmette-Guérin immunisation at birth and morbidity among Danish children: a prospective, randomised, clinical trial. Contemp Clin Trials 2015;42:213-18.

7 Sørup S, Benn CS, Poulsen A, et al. Live vaccine against measles, mumps, and rubella and the risk of hospital admissions for nontargeted infections. JAMA 2014;311:826-35.

8 Statens Serum Institut. The Danish childhood vaccination program. http://www.ssi. $\mathrm{dk} /$ accination/Boernevaccination/Boernevaccinationsprogrammet.aspx. 2-1-2015. 8-6-2015.Ref Type: Online Source.

9 Andersen TF, Madsen M, Jorgensen J, et al. The Danish National Hospital Register. A valuable source of data for modern health sciences. Dan Med Bull 1999;46: 263-8.

10 Lynge E, Sandegaard JL, Rebolj M. The Danish National Patient Register. Scand J Public Health 2011;39Suppl):30-3.

11 Kjøller M, Juel K, Kamper-Jørgensen F. Folkesundhedsrapporten. 2007. http://www. si-folkesundhed.dk/upload/kap_30_b\%C3\%B8rn.pdf

12 Aaby $P$, Jensen $H$, Samb B, et al. Differences in female-Male mortality after high-titre measles vaccine and association with subsequent vaccination with diphtheria-tetanus-pertussis and inactivated poliovirus: reanalysis of West African studies. Lancet 2003;361:2183-8.

13 Kleinnijenhuis J, Quintin J, Preijers F, et al. Bacille Calmette-Guerin induces NOD2-dependent nonspecific protection from reinfection via epigenetic reprogramming of monocytes. Proc Natl Acad Sci USA 2012;109:17537-42.

14 Aaby P, Benn CS. Saving lives by training innate immunity with bacille Calmette-Guerin vaccine. Proc Natl Acad Sci USA 2012;109:17317-18.

15 Kleinnijenhuis J, Quintin J, Preijers F, et al. BCG-induced trained immunity in NK cells: role for non-specific protection to infection. Clin Immunol 2014;155: 213-19.

16 Jensen KJ, Larsen N, Biering-Sørensen S, et al. Heterologous immunological effects of early BCG vaccination in low-birth-weight infants in Guinea-Bissau: a randomized-controlled trial. J Infect Dis 2015;211:956-67. 
17 Aaby $\mathrm{P}$, Martins $\mathrm{CL}$, Garly ML, et al. Measles vaccination in the presence or absence of maternal measles antibody: impact on child survival. Clin Infect Dis 2014;59:484-92.

18 Lalor MK, Ben-Smith A, Gorak-Stolinska P, et al. Population differences in immune responses to Bacille Calmette-Guerin vaccination in infancy. $J$ Infect Dis 2009;199:795-800.

19 Lalor MK, Floyd S, Gorak-Stolinska P, et al. BCG vaccination induces different cytokine profiles following infant BCG vaccination in the UK and Malawi. J Infect Dis 2011;204:1075-85.

20 Devereux G, Seaton A, Barker RN. In utero priming of allergen-specific helper T cells. Clin Exp Allergy 2001;31:1686-95.
21 Kaufman JS, Dolman L, Rushani D, et al. The contribution of genomic research to explaining racial disparities in cardiovascular disease: a systematic review. Am J Epidemiol 2015;181:464-72.

22 McLaren PJ, Carrington M. The impact of host genetic variation on infection with HIV-1. Nat Immunol 2015;16:577-83.

23 Newport MJ. The genetic regulation of infant immune responses to vaccination. Front Immunol 2015;6:18.

24 Thomsen SF, Duffy DL, Kyvik KO, et al. Genetic influence on the age at onset of asthma: a twin study. J Allergy Clin Immunol 2010;126 626-30. 